\title{
Stripe Phases in Layered Nickelates
}

\author{
K. RośCiszewski ${ }^{a}$ AND A.M. OleS ${ }^{a, b}$ \\ ${ }^{a}$ Marian Smoluchowski Institute of Physics, Jagiellonian University, W.S. Reymonta 4, PL-30-059 Kraków, Poland \\ ${ }^{b}$ Max-Planck-Institut für Festkörperforschung, Heisenbergstraße 1, D-70569 Stuttgart, Germany \\ To describe quasi two-dimensional nickelates we introduce an effective Hamiltonian for $e_{\mathrm{g}}$ electrons, which \\ includes the kinetic energy, on-site Coulomb interactions, spin-spin and Jahn-Teller (static) terms. The experi- \\ mental stripe phases are correctly reproduced by the model. The mechanisms responsible for stripe formation are \\ different than those reported in cuprates and manganites.
}

PACS: 75.25.Dk, 75.47.Lx, 75.10.Lp, 63.20.Pw

\section{Introduction}

The phenomenon of stripes occurs in doped transition metal oxides. In particular it was discovered and extensively studied in cuprates [1] and manganites [2], later in nickelates [3]. For the theoretical description of nickelates see for example [4-6].

Here we describe the phase situation in doped monolayer nickelates $\mathrm{La}_{2-x} \mathrm{Sr}_{x} \mathrm{NiO}_{4}$ within the framework of the effective model featuring only $\mathrm{Ni}$ sites renormalized by the presence of the surrounding oxygens. The model was previously used for the description of manganites [7]. At each site the local basis is given by two Wannier orbitals of $e_{\mathrm{g}}$ symmetry, i.e., $x^{2}-y^{2}$ (in short notation $x$ ) and $3 z^{2}-r^{2}$ (in short notation $z$ ) orbitals.

\section{Model}

The Hubbard-type Hamiltonian $\mathcal{H}$ for two $e_{\mathrm{g}}$ orbital states is defined on a finite $6 \times 6$ cluster (periodic boundary conditions) and consists of kinetic, crystal field, on-site Coulomb, spin and Jahn-Teller terms

$\mathcal{H}=H_{\text {kin }}+H_{\text {cr }}+H_{\text {int }}+H_{\text {spin }}+H_{\mathrm{JT}}$.

The kinetic part with anisotropic phase dependent hopping (according to the Koster-Slater rules) is

$$
\begin{aligned}
& H_{\mathrm{kin}}=-\frac{1}{4} t_{0} \sum_{\{i j\} \| a b, \sigma}\left[\left(3 d_{i x \sigma}^{\dagger} d_{j x \sigma}+d_{i z \sigma}^{\dagger} d_{j z \sigma}\right)\right. \\
& \left. \pm \sqrt{3}\left(d_{i x \sigma}^{\dagger} d_{j z \sigma}+d_{i z \sigma}^{\dagger} d_{j x \sigma}\right)\right] .
\end{aligned}
$$

The creation operators $d_{i \mu \sigma}^{\dagger}$ correspond to electron in orbital $\mu=x, z$, with spin $\sigma=\uparrow, \downarrow$ located at the site $i$. The $\{i, j\}$ runs over pairs of nearest neighbors.

The kinetic energy is supplemented by orbital splitting due to crystal field $H_{\mathrm{cr}}=\frac{1}{2} E_{z} \sum_{i \sigma}\left(n_{i z \sigma}-n_{i x \sigma}\right)$.

The $H_{\text {int }}$ and $H_{\text {spin }}$ stand for standard Hubbard type on-site Coulomb and exchange interactions (for electrons within degenerate $e_{\mathrm{g}}$ orbitals) used before for monolayer, bilayer and cubic perovskite manganites [7]; the Hubbard on-site Coulomb interaction is denoted as $U$, the Hund exchange interaction constant is $J_{\mathrm{H}}$.

The simplified Jahn-Teller part $H_{\mathrm{JT}}$ is

$$
\begin{aligned}
& H_{\mathrm{JT}}=g_{\mathrm{JT}} \sum_{i}\left(Q_{1 i}\left(2-n_{i x}-n_{i z}\right)+Q_{2 i} \tau_{i}^{x}+Q_{3 i} \tau_{i}^{z}\right) \\
& +\frac{1}{2} K \sum_{i}\left(2 Q_{1 i}^{2}+Q_{2 i}^{2}+Q_{3 i}^{2}\right),
\end{aligned}
$$

where the pseudo-spin operators, i.e., $\left\{\tau_{i}^{x}, \tau_{i}^{z}\right\}$ operators, are defined as follows: $\tau_{i}^{x}=\sum_{\sigma}\left(d_{i x \sigma}^{\dagger} d_{i z \sigma}+d_{i z \sigma}^{\dagger} d_{i x \sigma}\right)$ and $\tau_{i}^{z}=\sum_{\sigma}\left(d_{i x \sigma}^{\dagger} d_{i x \sigma}-d_{i z \sigma}^{\dagger} d_{i z \sigma}\right)$. The JT term includes three modes, $\left\{Q_{1 i}, Q_{2 i}, Q_{3 i}\right\}$ which denote standard $e_{\mathrm{g}}$ static deformations of the $i$-th octahedron. (Let us note that for dynamic JT effect each two neighboring Ni atoms share one oxygen in between and thus neighboring JT distortions are not independent.)

\section{Results and discussion}

We performed the Hartree-Fock computations and following that the electron correlations were treated by using local ansatz [8] (for detailed description see [7]). The correlations were found to be much stronger than those reported in cuprates and manganites. The phase situation for the doping levels $x=0, \frac{1}{3}, \frac{1}{2}$ was reproduced very well as shown in Figs. 1-3.

The diagonal stripe phases appear only for $x=\frac{1}{3}$ and $x=\frac{1}{2}$. They are not like the stripes in cuprates and manganites. In cuprates charge order coexists with the modulation of antiferro order between alternating domains. The domain boundaries are non-magnetic and half-filled. The mechanism of their formation is most probably a competition between kinetic energy and strong magnetic energy (superexchange). The Jahn-Teller interactions do not seem to be of importance. 


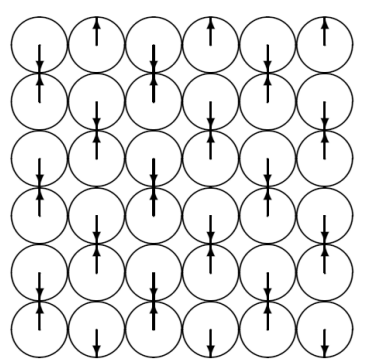

Fig. 1. G-AF antiferro (spin = 1) with uniform charge distribution ground-state phase as obtained for the doping $x=0$ (Hamiltonian parameters: $t_{0}, J_{\mathrm{H}}, E_{z}=0.6$, $0.9,-0.3 \mathrm{eV}, U=8 t_{0}, g_{\mathrm{JT}}=3.0 \mathrm{eV} / \AA, K=\mathrm{eV} / \AA^{2}$ ). No orbital order. Legend: at each site the circle diameter corresponds to one-half of $e_{\mathrm{g}}$ on-site charge; the arrow length to one-half of the $e_{\mathrm{g}}$ spin; the horizontal bar length (if present) to charge density difference between $x$ and $z$ orbitals (longest bar to the right corresponds to pure $x$, to the left to pure $z$, zero length to half by half combination). All these values are expressed in approximate proportionality to nearest neighbor site-site distance which is assumed to be unity.
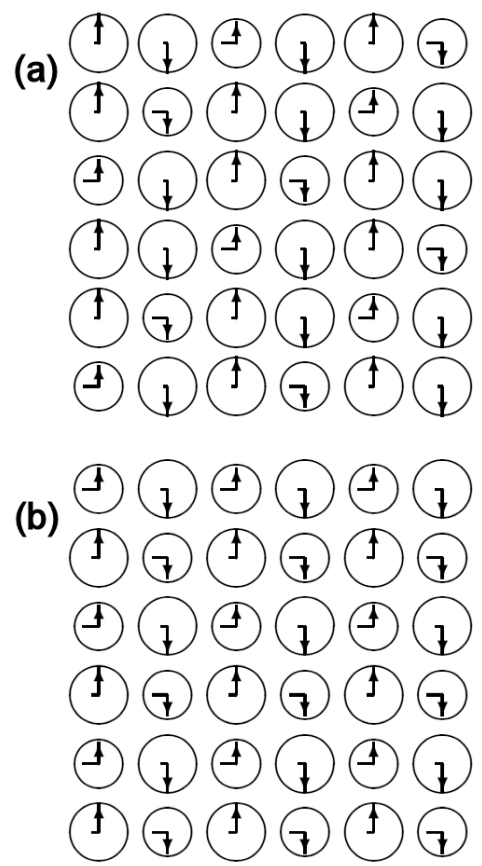

Fig. 2. (a) $C$-AF spin arrangement (ferromagnetic vertical lines coupled antiferomagnetically), with uniform charges on charge majority sites (spins $S \approx 1$ ), as obtained for the $x=1 / 3$. The charge minority sites form diagonal stripe boundaries (each third line). They are occupied predominantly by $z$ electrons. (b) Perfect checkerboard crystal-like order with two charge majority/minority sublattices as obtained for $x=1 / 2$ doping. Diagonal stripes - each second line. On average $C$-AF spin order. The holes are predominantly doped into $x$ orbitals (charge minority sites). Legend and the values of parameters as in Fig. 1.

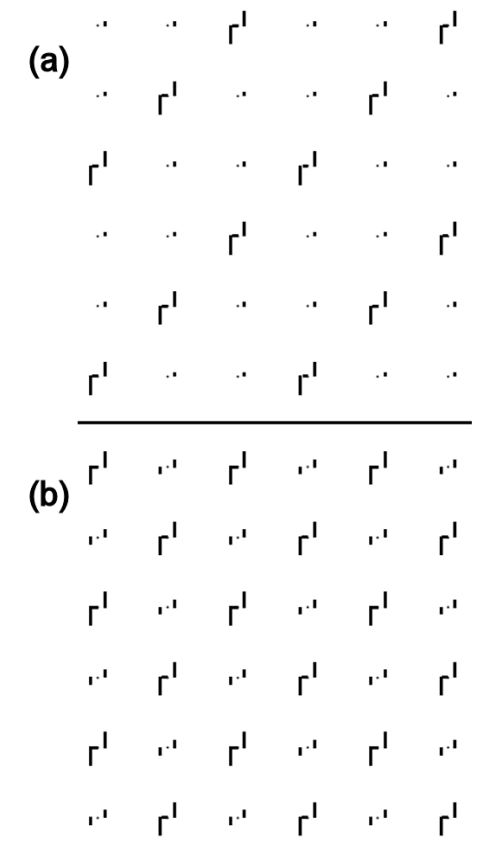

Fig. 3. Jahn-Teller distortions directly corresponding to the charge, spin and orbital order as shown in Fig. 2 (for $1 / 3$ and $1 / 2$ dopings). The distortions $Q_{2 i}$ (in $\AA$, scaled by a factor of 2) are shown as vertical bars drawn slightly to the left of each site and $Q_{3 i}$, by bars to the right of each site. Isotropic mode $Q_{1 i}$ is not present. Uniform electron distribution between $x$ and $z$ orbitals results in no JT distortions at charge majority sites, while non-zero JT distortions at charge minority sites stabilize the orbital order. Legend and the values of parameters as in Fig. 1.

In manganites the situation is more complex due to possible presence of orbital order and due to extra spinspin interaction of $e_{\mathrm{g}}$ electrons with core $t_{2 \mathrm{~g}}$ spins. There are many competing mechanisms entering on equal footing and the distinct stripe patterns with the true long range order are not a rule. Moreover, the magnetic order of the ground state was found to be very sensitive to the precise values of the model parameters. However, at the $x=\frac{1}{2}$ doping there is a clear tendency of monolayer manganites to undergo the transition into two charge majority and charge minority subsystems (see Ref. [7], the first entry). This can be either checkerboard or vertical alternating lines arrangement. Superimposed on the charge order is the magnetic $\mathrm{CE}$ (zig-zag) or $C$-AF order respectively (here we refer to the total spin due to $e_{\mathrm{g}}$ and $t_{2 \mathrm{~g}}$ electrons). Without the JT coupling the two phases are energetically very close one to another. The JT effect can stabilize the CE magnetic plus checkerboard charge order. (Then the JT distortions are present only on charge majority sites.) In summary the mechanism of inhomogeneous ground-state formation is again competition of electron kinetic energy versus magnetic superexchange. The secondary but still important role is played by electronic correlations and JT effect. 
The situation in layered nickelates is again different. The main difference is that in the undoped substance there are two $e_{\mathrm{g}}$ electrons per site. When the hole is doped onto an ion then in the site one electron remains. The non-zero Coulomb repulsion is still present and the site still participates in magnetic order. Moreover on the doped ions the JT distortions develop which stabilizes the hole positioning. In effect one can say that kinetic energy of the doped holes is suppressed and the competition between magnetic and kinetic energy is absent in a part. The local JT distortions seem to govern the stripe formation. Therefore at low dopings the static stripes do not form. Only at the doping $x=1 / 3$ when the doped sites can appear as next nearest neighbors the diagonal stripe (each third line) appear. Similarly for $x=1 / 2$ doping the diagonal stripes (each second line) appear. When the JT coupling is turned off the stripes do not form (not shown). Similar situation occurs if we assume that the on-site Coulomb repulsion in our effective model is stronger than the one assumed in above. For example if $U=12 t_{0}$ the stripes develop the same way as shown in Figs. 1-3.

\section{Conclusions}

The results of the present study uncover the differences between the stripes in cuprates and in nickelates. A competition between the magnetic energy and the kinetic energy of doped holes drives the stripe structures in doped cuprates.

In layered nickelates when hole doping occurs in $d^{8}$ configuration, the ions occupied by holes carry a spin $1 / 2$ and therefore still participate in the magnetic order. In addition the doped ions are Jahn-Telller active and local distortions form around them. Both these effects suppress the kinetic energy of the doped holes and the competition between the magnetic and kinetic energy turns out to be not too important.

It is the Jahn-Teller effect (in presence of strong local correlations) which is primarily responsible for the stripe phase which occurs only at the $x=1 / 3$ doping and for the checkerboard phase at the $x=1 / 2$ doping.

\section{Acknowledgments}

The financial support by the Polish Ministry of Science and Higher Education under project No. N202 104138 is acknowledged.

\section{References}

[1] S.A. Kivelson, I.P. Bindloss, E. Fradkin, V. Oganesyan, J.E. Tranquada, A. Kapitulnik, C. Howald, Rev. Mod. Phys. 75, 1201 (2003); M. Raczkowski, R. Frésard, A.M. Oleś, Low Temp. Phys. 32, 305 (2006); M. Vojta, Adv. Phys. 58, 699 (2009).

[2] E. Dagotto, T. Hotta, A. Moreo, Phys. Rep. 344, 1 (2001); T. Hotta, A. Feiguin, E. Dagoto, Phys. Rev. Lett. 86, 4922 (2001); E. Dagotto, New J. Phys. 7, 67 (2009).

[3] J.M. Tranquada, D.J. Buttrey, V. Sachan, J.E. Lorenzo, Phys. Rev. Lett. 73, 1003 (1994); V. Sachan, D.J. Buttrey, J.M. Tranquada, J.E. Lorentzo, G. Shirane, Phys. Rev. B 51, R12742 (1995); R. Kajimoto, K. Ishizaka, H. Yoshizawa, Y. Tokura, Phys. Rev. B 67, 014511 (2003); P.D. Spencer, M.E. Ghazi, S.B. Wilkins, P.D. Hatton, S.D. Brown, D. Prabhakaran, A.T. Boothroyd, Eur. Phys. J B 46, 27 (2005).

[4] T. Hotta, E. Dagotto, Phys. Rev. Lett. 92, 227201 (2004).

[5] M. Raczkowski, R. Frésard, A.M. Oleś, Phys. Rev. B 73, 094429 (2006).

[6] U. Schwingenschlögl, C. Schoster, R. Frésard, Europhys. Lett. 81, 27002 (2008); Europhys. Lett. 88, 67008 (2010).

[7] K. Rościszewski, A.M. Oleś, J. Phys.: Condens. Matter 19, 186223 (2007); J. Phys., Condens. Matter 20, 365212 (2008); J. Phys., Condens. Matter 22, 425601 (2010).

[8] G. Stollhoff, P. Fulde, J. Chem. Phys. 73, 4548 (1980); G. Stollhoff, J. Chem. Phys. 105, 227 (1996); P. Fulde, Electron Correlations in Molecules and Solids, Springer Series in Solid State Sciences, Vol. 100, Springer Verlag, Berlin 1991. 